\title{
New therapeutic strategy for amyloidosis
}

\section{Akiyoshi Morinaga, Kenjiro Ono \& Masahito Yamada ${ }^{\dagger}$ \\ †Author for correspondence: Department of Neurology \& Neurobiology of Aging, Kanazawa University Graduate School of Medical Science, Kanazawa 920-8640, Japan = Tel.: +81 762652292 \\ = Fax: +81 762344253 m m-yamada@med.kanazawa-u.ac.jp}

Evaluation of: Ozawa D, Yagi H, Ban T et al.: Destruction of amyloid fibrils of a $\beta_{2}$-microglobulin fragment by laser beam irradiation. J. Biol. Chem. 284(2), 1009-1017 (2009). In this study, the authors discovered that laser beam irradiation with thioflavin T could stop the extension of $\beta_{2}$-microglobulin fibrils and even degrade preformed amyloid fibrils. These reactions require both thioflavin T and a laser beam at $442 \mathrm{~nm}$; active oxygen plays an important role. This approach may be a new therapeutic strategy for destroying fibrils in dialysis-related amyloidosis as well as other types of amyloidosis or related disorders, including Alzheimer's disease, familial amyloid polyneuropathy, Parkinson's disease and Huntington's disease.

This article reviews the recent publication by Ozawa and colleagues [1]. Amyloidosis and amyloid-related disorders, including Alzheimer's disease (AD), Parkinson's disease (PD), Huntington's disease, familial amyloid polyneuropathy (FAP) and dialysis-related amyloidosis (DRA), are associated with extra- or intra-cellular deposition of fibrillar proteins [2]. Furthermore, amyloid-like fibrils can be formed with various proteins and peptides that are not related to diseases [2]. The major components of amyloid or amyloid-like fibrils include amyloid- $\beta(A \beta)$ protein in $A D, \alpha$-synuclein $(\alpha S)$ in $\mathrm{PD}$, transthyretin in FAP and $\beta_{2}$-microglobulin $\left(\beta_{2}-\mathrm{m}\right)$ in DRA [3]. During the development of disease-modifying therapies, many researchers favor approaches that target fibril formation, deposition and clearance.

Thioflavin T (ThT), benzothiazole and aminobenzene rings connected by a rotating single bond have been used as reagents to detect and measure the amount of amyloid fibrils. When ThT binds to amyloid fibrils, it fluoresces brightly at an excitation and emission of 440-450 and 480-490 $\mathrm{nm}$, respectively, and the brightness depends on the amount of amyloid fibrils. On the other hand, ThT does not show such fluorescence before fibril formation [4].

Previously, amyloid-specific ThT fluorescence was combined with total internal reflection fluorescence microscopy (TIRFM), which enables the direct observation of amyloid fibril growth [5]. TIRFM can selectively monitor fibrils and obtain their exact length and, hence, the kinetics of seed-dependent fibril growth can be analyzed by real-time analysis of amyloid fibrils.
A 22-residue K3 peptide of $\beta_{2}-\mathrm{m}\left(\mathrm{Ser}^{20}-\mathrm{Lys}^{41}\right)$ is one of the core regions of $\beta_{2}-\mathrm{m}$ amyloid fibrils and is thought to play a crucial role in the fibrillation process [6]. This K3 peptide spontaneously forms amyloid fibrils at neutral $\mathrm{pH}$ [7]. Furthermore, it has been reported that the K3 peptide forms amyloid fibrils over a wide range of $\mathrm{pH}$ and solvent conditions [7-9].

The fibrillation process of $\beta_{2}-\mathrm{m}$ and $\mathrm{K} 3$, which is a part of $\beta_{2}-\mathrm{m}$ and thought to play a core role in $\beta_{2}$-m fibrillation, has been examined using TIRFM [6]. Surprisingly, Ozawa and colleagues discovered that the laser with ThT could stop fibril extension and even degrade the preformed K3 fibrils.

\section{Summary of methods \& results}

Recombinant human $\beta_{2}$-m was expressed with an Escherichia coli expression system and purified as described previously [10]. Chemically synthesized K3 peptides were purchased from Peptide Institute, Inc. (Osaka, Japan). The surface of the slides was positively charged by the adsorption of polyethyleneimine (Nacalai tesque, Kyoto, Japan)/polyvinylsulfonate (Sigma Chemical Co., MO, USA) or negatively charged by layer-by-layer deposition of polyelectrolytes. Ozawa et al. mixed $25 \mu \mathrm{M} \beta_{2}-\mathrm{m}$ with $30 \mu \mathrm{g} / \mathrm{ml}$ seeds, prepared by sonication of $\beta_{2}$-m fibrils, or $100 \mu \mathrm{M} \mathrm{K} 3$ without seeds, with 5 and $10 \mu \mathrm{M}$ ThT, respectively, and these were incubated at $37^{\circ} \mathrm{C}$ on tightly covered microscopic slides. The slides were observed using the TIRFM system at an excitation wavelength of $442 \mathrm{~nm}$. The growth of $\beta_{2}$-m fibrils was independent of the properties of the slide surface, whereas the growth of K3 fibrils varied depending on the surface. The growth of K3 fibrils on
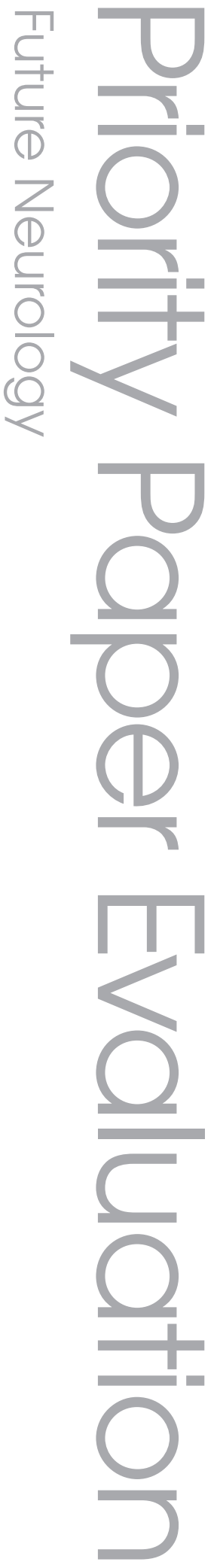

future medicine $^{\text {pisg }}$ fs 
the negatively charged surface was explosive, whereas that on the positively charged surface was less extensive.

Intermittent irradiation on $\mathrm{K} 3$ fibrils was performed to examine the effect of laser irradiation. After irradiating the sample solution at $442 \mathrm{~nm}$, $\beta_{2}-\mathrm{m}$ and $\mathrm{K} 3$ fibril growth were inhibited, and the destruction of preformed K3 fibrils was observed by morphological analysis (atomic force microscopy [Nanoscope IIIa scanning microscope; Veeco, Tokyo, Japan] and the TIRFM system) and quantitative analysis (fluorescence spectroscopy with ThT and light scattering). These results suggest that the above-mentioned reactions require both ThT and a laser beam at $442 \mathrm{~nm}$.

To examine the involvement of active oxygen in the destruction of $\mathrm{K} 3$ fibrils during the excitation of ThT, the samples were purged with oxygen or nitrogen under laser irradiation. The intensity of light scattering decreased at a much greater rate when the oxygen was purged, although it did not decrease significantly when nitrogen was purged. A singlet oxygen sensor green reagent (Molecular Probes/Invitrogen, CA, USA), which measures singlet oxygen by fluorescence analysis (excitation: $504 \mathrm{~nm}$; emission: $525 \mathrm{~nm}$ ), was used to detect the generation of active oxygen during the excitation of amyloid-bound ThT. The laserirradiated sample showed high fluorescence of the singlet oxygen sensor in the presence of ThT; furthermore, the fluorescence intensity increased when oxygen was purged, thus indicating the generation of singlet oxygen.

High-pressure liquid chromatography was performed to detect chemical damage to the monomer. The irradiated K3 fibrils solution, dissolved in 6M guanidine hydrochloride, decreased significantly in intensity, and produced multiple broad tailing peaks, thus indicating the existence of products of various molecular weights, other than the K3 monomer and dimer. Furthermore, mass spectroscopy analysis of irradiated K3 fibrils revealed several decomposed peaks as well as the monomer peak. These peaks also had a smaller molecular weight than the monomer, thus suggesting the cleavage of peptide bonds by the laser. Amino acid analysis was performed to address the chemical modification. Three hours after irradiation, the intensity of His was decreased to $40 \%$ of the reference sample without irradiation.

\section{Discussion \& significance}

The present study by Ozawa and colleagues showed that irradiation with a laser beam at $442 \mathrm{~nm}$ inhibited $\mathrm{K} 3$ and $\beta_{2}-\mathrm{m}$ fibril formation and broke preformed $\mathrm{K} 3$ fibrils into shorter fibrils and monomers. These reactions required the energy of amyloid-bound ThT, which was transferred to ground-state oxygen, thus producing singlet oxygen and oxygen radicals. The active oxygen and ThT may have attacked K3 fibrils. The decrease in the His peak suggested that Tyr and Cys residues were also damaged [11].

The mechanism of damage to K3 fibrils might be similar to that caused by photodynamic therapy (PDT), which is used to treat some cancers, such as lung, stomach, uterus and skin cancer. PDT is a disease site-specific treatment, requiring the local or systemic administration of a photosensitizer, followed by irradiation of the target lesion. Active oxygen also plays an important role, similar to that played by laser irradiation, in PDT [12]. In PDT, active oxygen, including singlet oxygen, shows cytotoxic effects to tumor cells and vessels $[12,13]$. When these irradiation effects are applied to amyloidoses, fine tuning of oxidative stress will be necessary to destroy amyloid fibrils and to prevent injury to normal tissue. As the authors point out, a relatively weak laser irradiation accelerates $A \beta$ fibril growth, whereas extensive irradiation destroys $A \beta$ fibrils. Oxidative stress is closely related to pathogenesis of $\mathrm{AD}$ [14].

If the effects of oxidative stress can be fine tuned, then photosensitizers and light delivery systems must be developed for clinical application. Some amyloid imaging probes, such as PIB (PittsburgCompound B; $\left.\left[{ }^{11} \mathrm{C}\right] 6-\mathrm{OH}-\mathrm{BTA}-1\right)$, FDDNP (2-(1-\{6-[(2-[F-18]fluoroethyl)(methyl)amino]2-naphthyl\}ethylidene) malononitrile) and SB-13 (4-N-methylamino-4'-hydroxystilbene), have been recently developed for in vivo positron emission studies and have been evaluated in humans [15]. These amyloid-specific molecules have a structure similar to ThT and some are able to cross the blood-brain barrier and, hence, they are likely to be helpful for developing photosensitizers. During PDT for cancer, light can be directly delivered to the focal area by a bronchoscope, gastroscope or colposcope. However, it is generally difficult to focus light on amyloid-deposited organs or tissues (brain, nerve, heart, kidney and joint); amyloid fibrils are diffusely distributed in the target organ or tissues. Therefore, a new idea is necessary to develop the light delivery systems.

\section{Conclusion \& future perspective}

Ozawa and colleagues discovered that the laser with ThT could stop $\beta_{2}$-m fibril extension and even degrade preformed K3 fibrils, which may provide a new therapeutic approach to amyloidosis and related disorders. The clarification of this irradiation effect as well as 
the development of effective photosensitizers and light delivery systems is necessary for a successful clinical application.

\section{Financial \& competing interests disclosure}

The authors have no relevant affiliations or financial involvement with any organization or entity with a financial interest in or financial conflict with the subject matter or materials discussed in the manuscript. This includes employment, consultancies, honoraria, stock ownership or options, expert testimony, grants or patents received or pending, or royalties.

No writing assistance was utilized in the production of this manuscript

\section{Executive summary}

\section{Background}

- Prevention of amyloid fibril formation and the clearance of amyloid are attractive therapeutic approaches for amyloidosis.

- $\beta_{2}$-microglobulin $\left(\beta_{2}-m\right)$ is a major component of amyloid fibrils deposited in patients with dialysis-related amyloidosis, and a 22-residue K3 fragment of $\beta_{2}-m$ is one of the core regions of the fibrils.

\section{Methods \& results}

- After irradiation with thioflavin T (ThT), $\beta_{2}-\mathrm{m}$ and $\mathrm{K} 3$ fibril growth were inhibited, and the destruction of preformed K3 fibrils was observed by morphological and quantitative analyses.

- These reactions require the energy of amyloid-bound ThT, which is transferred to ground-state oxygen, thus producing singlet oxygen and oxygen radicals.

\section{Discussion \& significance}

- Anti-amyloid effects of laser beam irradiation and ThT could form a new therapeutic approach to dialysis-related amyloidosis and other amyloidoses.

- Amyloid imaging probes with a similar structure to ThT might be useful as photosensitizing agents in this new therapeutic strategy.

\section{Conclusion \& future perspective}

- The authors have suggested a new therapeutic strategy for amyloidosis.

\section{Bibliography}

1. Ozawa D, Yagi H, Ban T et al.: Destruction of amyloid fibrils of a $\beta_{2}$-microglobulin fragment by laser beam irradiation. J. Biol. Chem. 284(2), 1009-1017 (2009).

2. Dobson CM: Protein folding and misfolding. Nature 426, 884-890 (2003).

3. Lashuel HA, Lansbury PT Jr: Are amyloid diseases caused by protein aggregates that mimic bacterial pore-forming toxins? Q. Rev. Biophys. 39(2), 167-201 (2006)

4. Naiki H, Higuchi K, Nakakuki K, Takeda T: Kinetic analysis of amyloid fibril polymerization in vitro. Lab. Invest. 65(1), 104-110 (1991).

5. Ban T, Hamada D, Hasegawa K, Naiki H, Goto Y: Direct observation of amyloid fibril growth monitored by thioflavin $\mathrm{T}$ fluorescence. J. Biol. Chem. 278(19), 16462-16465 (2003).

6. Kozhukh GV, Hagihara Y, Kawakami T, Haseawa K, Naiki H, Goto Y: Investigation of a peptide responsible for amyloid fibril formation of $\beta_{2}$-microglobulin by Achromobacter Protease I. J. Biol. Chem. 277(2), 1310-1315 (2002).

7. Ohhashi Y, Hasegawa K, Naiki H, Goto Y: Optimum amyloid fibril formation of a peptide fragment suggests the amyloidogenic preference of $\beta_{2}$-microglobulin under physiological conditions. J. Biol. Chem. 279(11), 10814-10821 (2004).
8. Iwata K, Fujiwara T, Naiki $\mathrm{H}$ et al: $3 \mathrm{D}$ structure of amyloid protofilaments of $\beta_{2}$-microglobulin fragment probed by solid-state NMR. Proc. Natl Acad. Sci. USA 103(48), 18119-18124 (2006).

9. Yamaguchi K, Takahashi S, Kawai T, Naiki H, Goto Y: Seeding-dependent propagation and maturation of amyloid fibril conformation. J. Mol. Biol. 352(4), 952-960 (2005).

10. Chiba T, Hagihara Y, Higurashi T, Hasegawa K, Naiki H, Goto Y: Amyloid fibril formation in the context of full-length protein: effects of proline mutations on the amyloid fibril formation of $\beta_{2}$-microglobulin. J. Biol. Chem. 278(47), 47016-47024 (2003).

11. Davies MJ: Reactive species formed on proteins exposed to singlet oxygen. Photochem. Photobiol. Sci. 3(1), 17-25 (2004).

12. Huang Z, Xu H, Myeyers AD et al.: Photodynamic therapy for treatment of solid tumors - potential and technical challenges. Technol. Cancer Res. Treat. 7(4), 309-320 (2008).

13. Henderson BW, Dougherty TJ: How does photodynamic therapy work? Photochem. Photobiol. 55(1), 145-157 (1992).

14. Bieschke J, Zhang Q, Bosco DA et al:: Small molecule oxidation products trigger diseaseassociated protein misfolding. Acc. Chem. Res. 39(9), 611-619 (2006).

15. Wu C, Pike VW, Wang Y: Amyloid imaging: from bench top to bedside. Curr. Top. Dev. Biol. 70, 171-213 (2005).

\section{Affiliations}

- Akiyoshi Morinaga

Department of Neurology \& Neurobiology of Aging, Kanazawa University Graduate School of Medical Science, Kanazawa, 920-8640, Japan

Tel.: +81762652292

Fax: +81762344253

amorinag@med.kanazawa-u.ac.jp

- Kenjiro Ono

Department of Neurology \& Neurobiology of Aging, Kanazawa University Graduate School of Medical Science, Kanazawa, 920-8640, Japan

and,

Department of Neurology, David Geffen School of Medicine \& Molecular Biology Institute \& Brain Research Institute, University of California, Los Angeles, CA 90095, USA

Tel.: +81762652292

Fax: +81762344253

onoken@med.kanazawa-u.ac.jp

- Masahito Yamada

Department of Neurology \& Neurobiology of Aging, Kanazawa University Graduate School of Medical Science, Kanazawa 920-8640, Japan

Tel.: +81762652292

Fax: +81 762344253

m-yamada@med.kanazawa-u.ac.jp 